\title{
Verifying Lossy Channel Systems has Nonprimitive Recursive Complexity
}

\author{
$\mathrm{Ph}$. Schnoebelen \\ Lab. Spécification et Vérification \\ ENS de Cachan \& CNRS UMR 8643 \\ 61, av. Pdt. Wilson, \\ 94235 Cachan Cedex France \\ email: phs@lsv.ens-cachan.fr
}

Keywords: verification of infinite-state systems, communication protocols, program correctness, formal methods.

\begin{abstract}
Lossy channel systems are systems of finite state automata that communicate via unreliable unbounded fifo channels. It is known that reachability, termination and a few other verification problems are decidable for these systems. In this article we show that these problems cannot be solved in primitive recursive time.
\end{abstract}

\section{Introduction}

Channel systems, also called Finite State Communicating Machines, are systems of finite state automata that communicate via asynchronous unbounded fifo channels [Boc78, BZ83]. Figure 1 displays an example, where the labels $c ! x$ and $c ? x$ mean that message $x$ (a letter) is sent to (respectively read from) channel $c$. Channel systems are a natural model for asynchronous

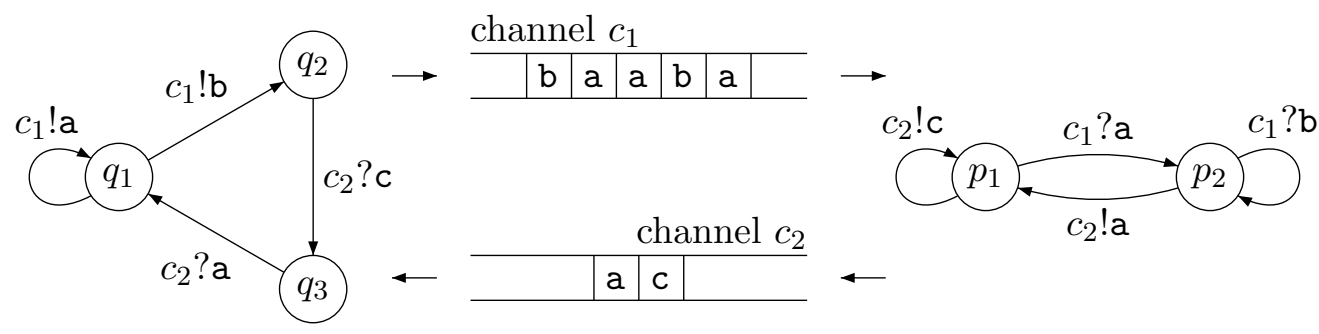

Figure 1: A channel system with two automata and two channels

communication protocols and constitute the semantical basis for ISO protocol specification languages such as SDL and Estelle. Channel systems are Turing powerful, and no verification method for them can be general and fully algorithmic.

A few years ago, Abdulla and Jonsson identified lossy channel systems as a very interesting model: in lossy channel systems messages can be lost while they are in transit, without 
any notification. These systems are very close to the completely specified protocols independently introduced by Finkel, and for which he showed the decidability of termination. Abdulla and Jonsson showed that reachability, safety properties over traces, and eventuality properties over states are decidable for lossy channel systems. The decidability results of [Fin94, CFP96, AJ96b] are fundamental since lossy systems are the natural model for fault-tolerant protocols where the communication channels are not supposed to be reliable (see [AKP97, ABJ98, AAB99] for applications).

For lossy channel systems, the aforementioned decidability results lead to algorithms whose termination rely on Higman's Lemma (see [AČJT00, FS01] for more examples of this phenomenon). No complexity bound is known and, e.g., Abdulla and Jonsson stated in [AJ96b] that they could not evaluate the cost of their algorithm.

In this article we show that all the above-mentioned decidable problems have nonprimitive recursive complexity, i.e., cannot be solved by algorithms with running time bounded by a primitive recursive function of their input size. This puts these problems among the hardest decidable problems.

Our proof relies on a simple construction showing how lossy channel systems can weakly compute some fast growing number-theoretic functions $A_{2}, A_{3}, \ldots$ related to Ackermann's function and their inverses $A_{2}^{-1}, A_{3}^{-1}, \ldots$ By "weakly computing $f$ " we mean that, starting from $x$, all values between 0 and $f(x)$ can be obtained. This notion was used by Rabin in his proof that equality of the reachability sets of Petri nets is undecidable, a proof based on the weak computability of multivariate polynomials (see [Hac76]). Petri nets can weakly compute the $A_{n}$ functions (see [MM81]) but they cannot weakly compute their inverses $A_{n}^{-1}$ as lossy channel systems can do.

There exist other families of systems that can weakly compute both $A_{n}$ and $A_{n}^{-1}$ : the lossy counter machines of [May00], or the reset nets of [DFS98, DJS99]. Our construction can easily be adapted to show that, for these systems too, decidable problems like termination, control-state reachability, ..., are nonprimitive recursive.

Finally, let us observe that there does not exist many uncontrived problems that have been shown decidable but not primitive recursive. In the field of verification, we are only aware of one instance: the "finite equivalence problem for Petri nets" ${ }^{1}$ introduced by Mayr and Meyer [MM81]. This problem is, given two Petri nets, to decide whether they both have the same set of reachable markings and this set is finite (equivalence is undecidable without the finiteness assumption). It can be argued that the verification (termination or reachability) of lossy channel systems is a less contrived problem.

\section{Channel systems, from perfect to lossy}

A channel system usually combines several finite-state automata that communicate through several channels. Here, and without loss of generality, we assume our systems only have one automaton that uses its several channels as fifo buffers.

\footnotetext{
${ }^{1}$ See [Jan01] for a more general proof that all finite equivalence problems for Petri nets are nonprimitive recursive.
} 
Formally, a channel system is a tuple $S=\langle Q, C, \Sigma, \Delta\rangle$ where $Q=\{q, \ldots\}$ is a finite set of control states, $C=\left\{c_{1}, \ldots, c_{k}\right\}$ is a finite set of channels, $\Sigma=\{\mathrm{a}, \mathrm{b}, \ldots\}$ is a finite alphabet of messages, and $\Delta \subseteq Q \times C \times\{?, !\} \times \Sigma^{*} \times Q$ is a finite set of transition rules (see below).

A configuration of $S$ is a tuple $\gamma=\left\langle q, w_{1}, \ldots, w_{k}\right\rangle$ denoting that control is currently in state $q$, while channels $c_{1}$ to $c_{k}$ contain words $w_{1}, \ldots, w_{k}$ (from $\Sigma^{*}$ ).

The transition rules in $\Delta$ state how $S$ can move from a configuration to another. Formally, $S$ has a "perfect" step $\gamma \rightarrow$ perf $\gamma^{\prime}$ iff $(1) \gamma$ is some $\left\langle q, w_{1}, \ldots, w_{k}\right\rangle,(2) \gamma^{\prime}$ is some $\left\langle q^{\prime}, w_{1}, \ldots, w_{i-1}, v, w_{i+1}, \ldots, w_{k}\right\rangle$, and (3.1) there is a rule $\left(q, c_{i}, !, u, q^{\prime}\right) \in \Delta$ such that $v=w_{i} u$ ( $u$ has been written to the tail of $c_{i}$ ) or $(3.2)$ there is a rule $\left(q, c_{i}, ?, u, q^{\prime}\right) \in \Delta$ such that $w_{i}=u v$ ( $u$ has been read from the head of $c_{i}$ ). These steps are called perfect because no message is lost.

It is well known that, assuming perfect steps, channel systems can faithfully simulate Turing machines in quadratic time [BZ83] (a single channel is enough to replace a Turing machine work tape; reading and writing in the middle of the channel requires rotating the content of the channel for positioning reasons, hence the quadratic overhead). Thus all interesting verification problems are undecidable for systems with perfect channels, even when restricted to single-channel systems.

\subsection{Lossy systems}

The most elegant and convenient way to model lossy channel systems is to see them as channel systems with an altered notion of steps [AJ96b].

We write $u \sqsubseteq v$ when $u$ is a subword of $v$, i.e. $u$ can be obtained by deleting any number

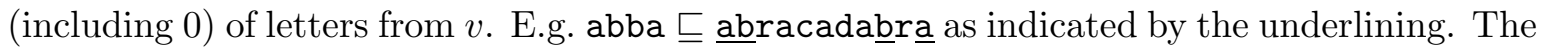
subword ordering extends to configurations: $\left\langle q, w_{1}, \ldots, w_{k}\right\rangle \sqsubseteq\left\langle q^{\prime}, v_{1}, \ldots, v_{k}\right\rangle \stackrel{\text { def }}{\Leftrightarrow} q=q^{\prime}$ and $w_{i} \sqsubseteq v_{i}$ for all $i=1, \ldots, k$. By Higman's Lemma [Hig52], this gives a a well-quasi-ordering:

Lemma 2.1 Every infinite sequence $\gamma_{0}, \gamma_{1}, \gamma_{2}, \ldots$ of configurations contains an infinite increasing subsequence $\gamma_{i_{0}} \sqsubseteq \gamma_{i_{1}} \sqsubseteq \gamma_{i_{2}} \sqsubseteq \cdots$ (with $\left.i_{0}<i_{1}<i_{2}<\cdots\right)$.

When $\gamma^{\prime} \sqsubseteq \gamma$ we write $\gamma \rightsquigarrow \gamma^{\prime}$ and say that $S$ may evolve from $\gamma$ to $\gamma^{\prime}$ by losing messages. The steps of a lossy channel system are all $\gamma \rightarrow \gamma^{\prime}$ s.t. $\gamma \rightsquigarrow \delta \rightarrow$ perf $\delta^{\prime} \rightsquigarrow \gamma^{\prime}$ for some configurations $\delta, \delta^{\prime}$ (i.e. losses may occur before and after a perfect step is performed).

Note that a perfect step is a special case of a lossy step. A run is a sequence $\gamma_{0} \rightarrow \gamma_{1} \rightarrow$ $\gamma_{2} \cdots$ of chained lossy steps. A perfect run is a run that uses perfect steps only. We use $\gamma \stackrel{*}{\rightarrow} \gamma^{\prime}$ and $\gamma \stackrel{*}{\rightarrow}_{\text {perf }} \gamma^{\prime}$ to denote the existence of a finite run (resp. perfect run) that goes from $\gamma$ to $\gamma^{\prime}$.

We are interested in the following two problems:

Termination: Given a channel system $S$ and an initial configuration $\gamma_{0}$, are all runs from $\gamma_{0}$ finite?

Reachability: Given a channel system $S$ and two configurations $\gamma_{0}$ and $\gamma_{f}$, is there a run from $\gamma_{0}$ to $\gamma_{f}$ ?

Theorem 2.2 [Fin94, AJ96b]. Termination and reachability are decidable for lossy channel systems.

In the remaining of this note we show 
Theorem 2.3 Termination and reachability for lossy channel systems have nonprimitive recursive complexity.

Theorem 2.3 also applies to the other verification problems that are known decidable for lossy channel systems. Indeed, termination is an instance of inevitability (shown decidable in [AJ96b]). Reachability is easily reduced to control-state reachability (shown decidable in [AJ96b]). Finally, termination can be reduced to simulation with a finite-state system ${ }^{2}$ (shown decidable in [AK95, AČJT00]). Thus we are entitled to claim that "verifying lossy channel systems has nonprimitive recursive complexity". Note that there exist many undecidable problems for lossy channel systems [AK95, CFP96, AJ96a, May00, ABPJ00, Sch01].

\section{The main construction}

\subsection{Ackermann's function}

Let $\left(A_{n}\right)_{n \geq 2}$ be the following sequence of functions over the natural numbers:

$$
\begin{aligned}
& A_{2}(k) \stackrel{\text { def }}{=} 2 k \\
& A_{n}(k) \stackrel{\text { def }}{=} \underbrace{A_{n-1} \circ \cdots \circ A_{n-1}}_{k \text { times }}(1) \quad \text { if } n>2 .
\end{aligned}
$$

Thus $A_{n}(0)=1$ for all $n>2$, and $A_{2}$ is followed by

$$
\begin{aligned}
& A_{3}(k)=2^{k} \\
& A_{4}(k)=2^{2^{\cdots}}
\end{aligned}
$$$$
\text { (a tower of } k 2 \text { 's) }
$$

and so on. The $A_{n}$ 's are monotonic and expansive in the following sense: for any $n \geq 2$ and $k \geq 0$

$$
A_{n}(k) \geq k, \quad A_{n}(k+1) \geq A_{n}(k), \quad A_{n+1}(k) \geq A_{n}(k) .
$$

We define inverse functions $\left(A_{n}^{-1}\right)_{n \geq 2}$ via

$$
A_{n}^{-1}(m) \stackrel{\text { def }}{=} \max \left\{k \mid A_{n}(k) \leq m\right\} .
$$

Observe that the $A_{n}^{-1}$ 's are partial functions. Another way to understand these functions is to notice that

$$
A_{n}^{-1}(m)=k \text { iff } \underbrace{A_{n-1}^{-1} \circ \cdots \circ A_{n-1}^{-1}}_{k \text { times }}(m)=1 .
$$

There exists many versions of Ackermann's function. One possible definition is $A c k(n) \stackrel{\text { def }}{=}$ $A_{n}(2)$. It is well-known that $A c k(n)$ dominates any primitive recursive function of $n$. Thus it follows from classical complexity-theoretic results that halting problems for Turing machines running in time or space bounded by $\operatorname{Ack}(n)$ ( $n$ being the size of the input) cannot be decided in primitive recursive time or space.

\footnotetext{
${ }^{2} S$ terminates iff it is not simulation-equivalent with a simple loop.
} 


\subsection{Weakly computing $A_{n}$ with expanders}

We construct a family $E_{2}, E_{3}, \ldots$ of expanders, channel systems that weakly compute $A_{2}(k)$, $A_{3}(k), \ldots$ As illustrated in Fig. $2, E_{n}$, the $n$th expander, uses $n$ channels: $c_{1}$ is the "output

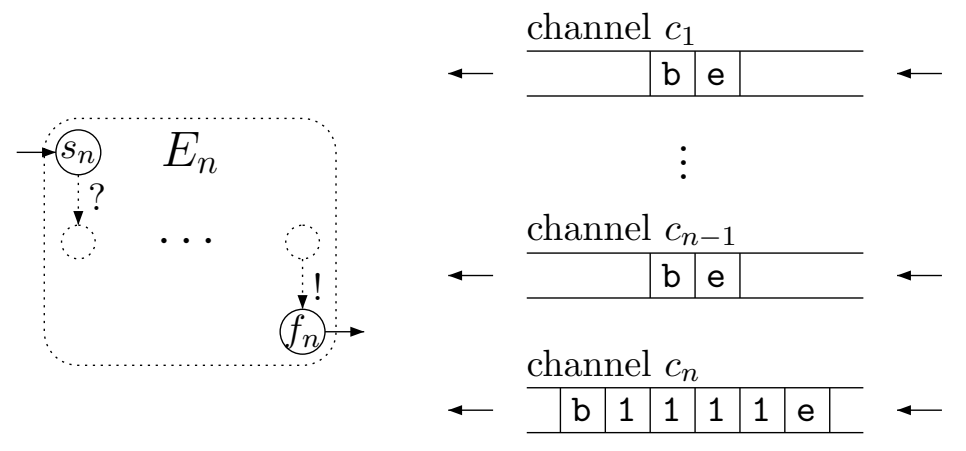

Figure 2: Interface for expander $E_{n}$

channel" (in which the system will write the result $A_{n}(k)$ ), $c_{n}$ is the "input channel" (from which the argument $k$ is read), and channels $c_{2}$ to $c_{n-1}$ are used to store auxiliary results. $E_{n}$ has one starting and one ending state, called $s_{n}$ and $f_{n}$ respectively.

These systems use a simple encoding for numbers: $k \in \mathbb{N}$ is encoded as a string $\lceil k\rceil$ over the alphabet $\Sigma_{0} \stackrel{\text { def }}{=}\{1, \mathrm{~b}, \mathrm{e}\}$. Formally, $\lceil k\rceil \stackrel{\text { def }}{=} \mathrm{b} 1^{k} \mathrm{e}$ is made of $k$ letters " 1 " surrounded by one "b" egin and one "e" nd marker. For example, the channels in Fig. 2 contain respectively $\lceil 0\rceil, \ldots,\lceil 0\rceil$, and $\lceil 4\rceil$.

Before explaining the construction of the expanders, we describe the simple transferring devices $T_{2}, T_{3}, \ldots$ they contain. This illustrates the way encodings $\lceil k\rceil$ of numbers are used. The $T_{n}$ 's, defined in Fig. 3 , start in state $t_{n}$ and end in state $x_{n}$ after they have transfered the contents of channel $c_{1}$ into channel $c_{n}$ (assuming $c_{1}$ contains at least $\lceil 1\rceil$ ). In Fig. 3 (and in future constructions) we sometimes omit depicting all intermediate states when their names are not required in the proof.

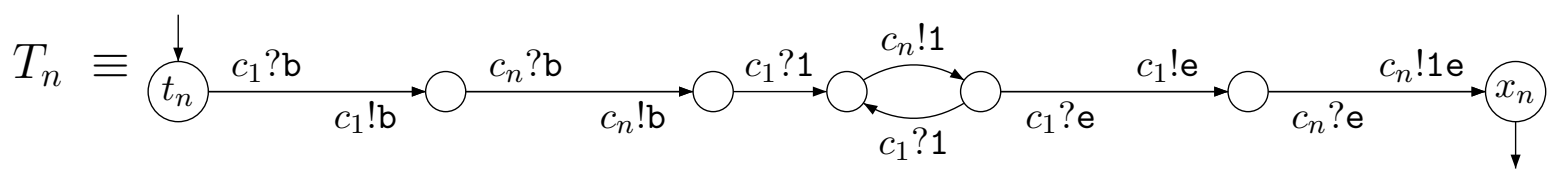

Figure 3: $T_{n}$, channel system for transferring $c_{1}$ into $c_{n}(n>1)$

Formally, the lossy behaviors of $T_{n}$ can be characterized by:

Proposition 3.1 For all $n \geq 2$,

$$
\left\langle t_{n},\left\lceil a_{1}\right\rceil, \ldots,\left\lceil a_{n}\right\rceil\right\rangle \stackrel{*}{\rightarrow}\left\langle x_{n},\left\lceil b_{1}\right\rceil, \ldots,\left\lceil b_{n}\right\rceil\right\rangle \text { iff }\left\{\begin{array}{l}
a_{1} \geq 1, b_{1}=0 \\
b_{i} \leq a_{i} \text { for } 1<i<n, \text { and } \\
b_{n} \leq a_{1}
\end{array}\right.
$$

Proof. Omitted. 
Note that the design of $T_{n}$ ensures that it blocks when $c_{1}$ contains $\lceil 0\rceil$. This property is used in the proof of Lemma 3.4.

We now move to the expanders themselves. The internals of $E_{n}$ are given in Fig. 4.

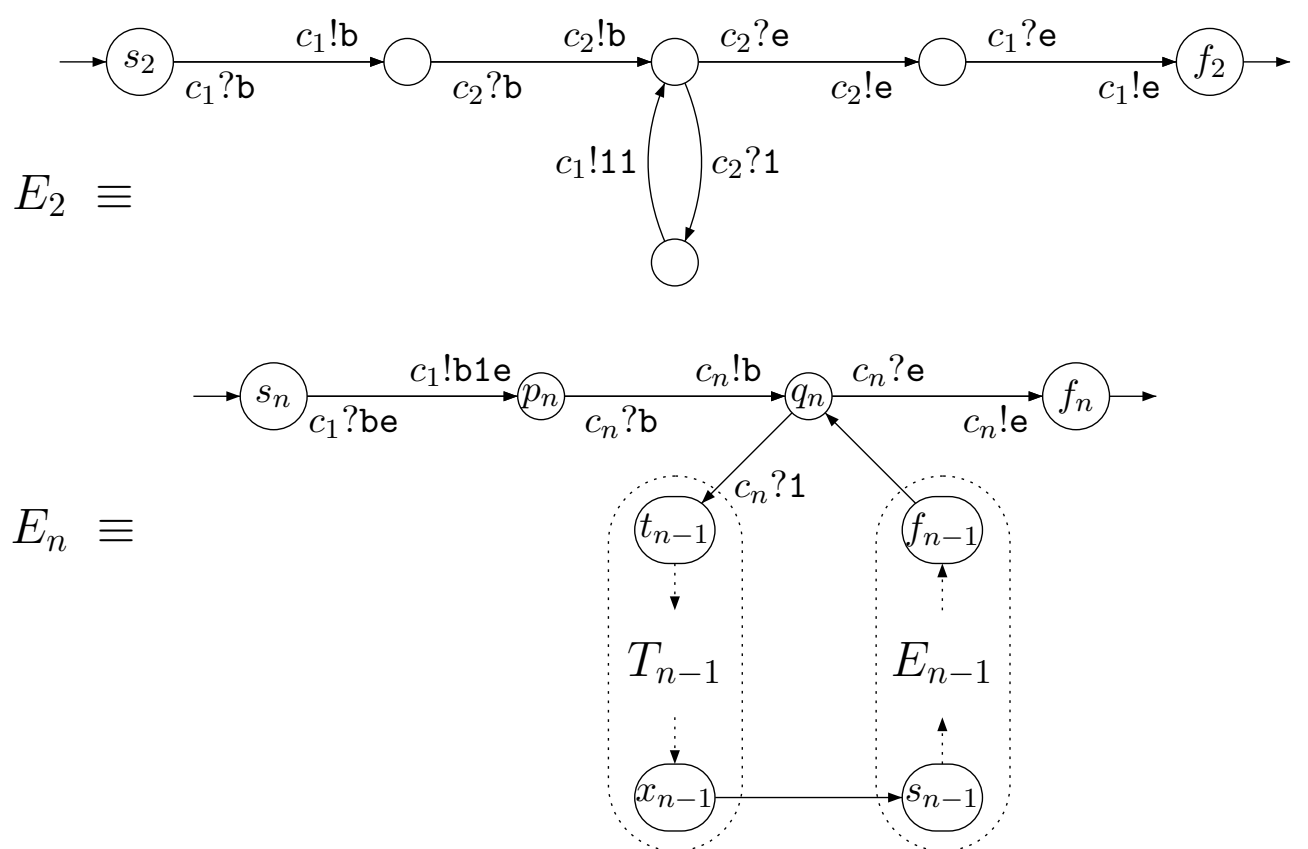

Figure 4: Expanders $E_{2}, E_{3}, \ldots$

For $n>2, E_{n}$ contains $E_{n-1}$ and $T_{n-1}$ as subsystems. This is because $E_{n}$ implements equation (2): every time a 1 is consumed from $c_{n}, E_{n-1}$ is run and the result is transfered (by $T_{n-1}$ ) from $c_{1}$ to $c_{n-1}$ so that $E_{n-1}$ can be applied again.

It is easy to convince oneself that the perfect (non-lossy) behavior of $E_{n}$ is to compute $A_{n}$ in the following formal sense:

$$
\left\langle s_{n},\lceil 0\rceil, \ldots,\lceil 0\rceil,\lceil k\rceil\right\rangle \stackrel{*}{\rightarrow}_{\operatorname{perf}}\left\langle f_{n}, w_{1}, \ldots, w_{n}\right\rangle \text { iff }\left\{\begin{array}{c}
w_{1}=\left\lceil A_{n}(k)\right\rceil \\
\text { and } \\
w_{2}=\ldots=w_{n}=\lceil 0\rceil .
\end{array}\right.
$$

Indeed, for $n>2$ a perfect run has the following form (where configurations are displayed in 
vector notation):

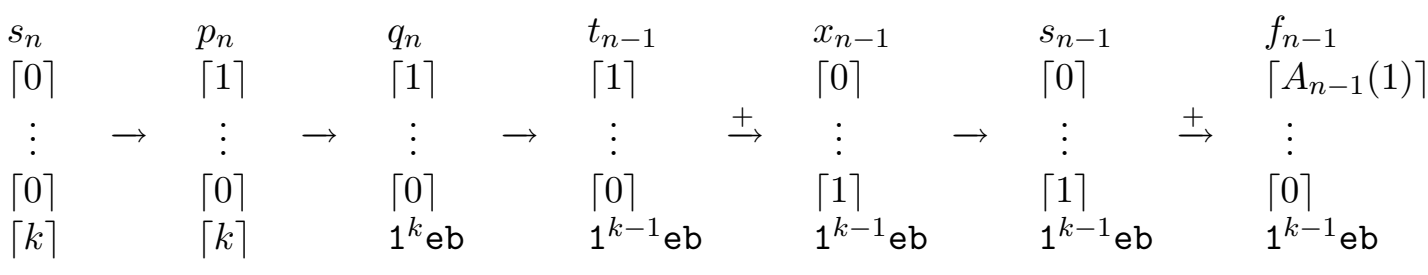

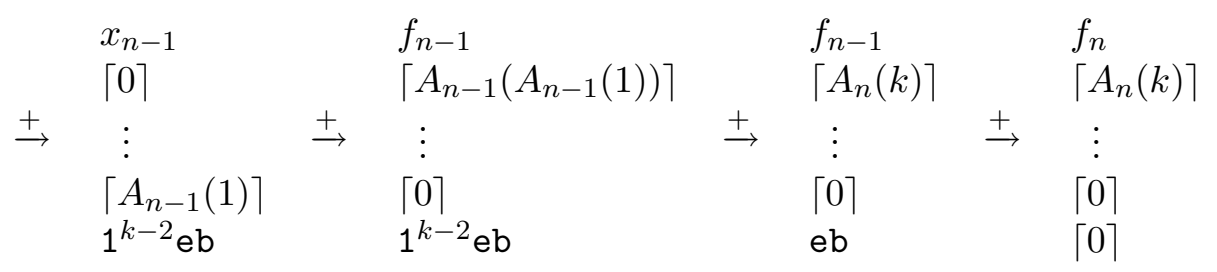

When perfect behavior is not assumed, $E_{n}$ still computes $A_{n}$, this time in a weak sense, according to the following statement:

Proposition 3.2 For all $n \geq 2$,

$$
\left\langle s_{n},\lceil 0\rceil, \ldots,\lceil 0\rceil,\lceil k\rceil\right\rangle \stackrel{*}{\rightarrow}\left\langle f_{n},\left\lceil a_{1}\right\rceil, \ldots,\left\lceil a_{n}\right\rceil\right\rangle \text { iff }\left\{\begin{array}{l}
a_{1} \leq A_{n}(k) \\
\text { and } \\
a_{2}=\ldots=a_{n}=0 .
\end{array}\right.
$$

Proof. The " $\Leftarrow$ " direction is an easy consequence of $(9)$. A detailed proof of the " $\Rightarrow$ " direction can be found in the Appendix but the underlying idea is simple: First, if some 1s are lost at any time during the computation, the final result will end up being smaller because of the monotonicity properties (5). Then, if $\mathrm{a} \mathrm{b}$ or a e marker is lost, the system can never recover it and will fail to reach a configuration where all channels contain encodings of numbers.

Note that the difference between (9) and (10) does not only come from the replacement of a " $=A_{n}(k)$ " by a " $\leq A_{n}(k)$ ": (9) guarantees that the $w_{i}$ s are encodings of numbers, while (10) assumes this.

\subsection{Weakly computing $A_{n}^{-1}$ with folders}

Folder systems $F_{2}, F_{3}, \ldots$ are channel systems that weakly compute the $A_{n}^{-1}$ 's. Here channel $c_{1}$ is the input channel and $c_{n}$ is the output, while channels $c_{2}$ to $c_{n-1}$ store auxiliary results. The definition of the $F_{n}$ 's, given in Fig. 5 , is based on (7). It uses transferring systems $T_{n}^{\prime}$ : these systems are variants of the $T_{n}$ 's and move the contents of channel $c_{n}$ into channel $c_{1}$ (instead of the other way around).

When possibly lossy behaviors are considered, $F_{n}$ weakly computes $A_{n}^{-1}$ in the following formal sense:

Proposition 3.3 For all $n \geq 2$,

$$
\left\langle s_{n}^{\prime},\lceil m\rceil,\lceil 0\rceil, \ldots,\lceil 0\rceil\right\rangle \stackrel{*}{\rightarrow}\left\langle f_{n}^{\prime},\left\lceil a_{1}\right\rceil, \ldots,\left\lceil a_{n}\right\rceil\right\rangle \text { iff }\left\{\begin{array}{l}
A_{n}\left(a_{n}\right) \leq m, \text { and } \\
a_{1}=\ldots=a_{n-1}=0 .
\end{array}\right.
$$




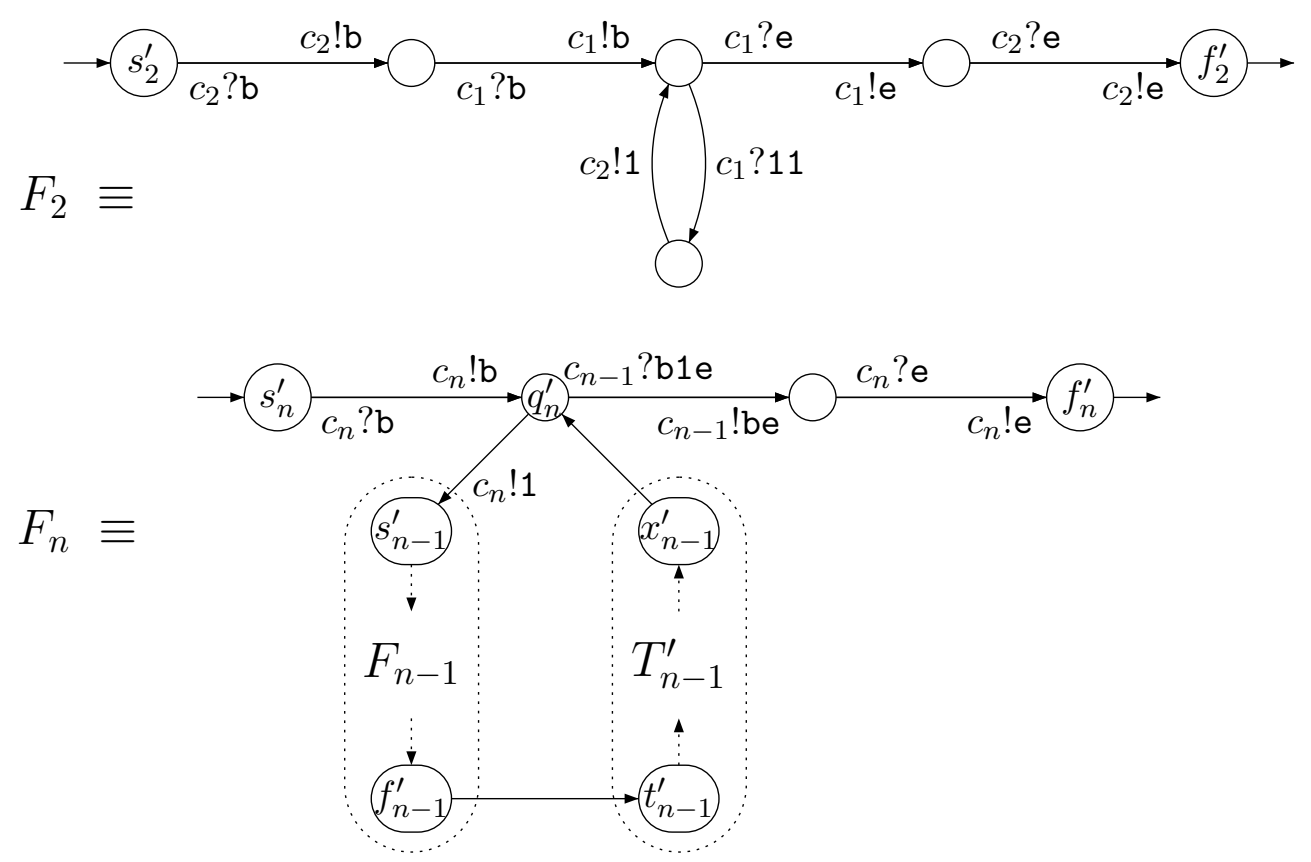

Figure 5: Folders $F_{2}, F_{3}, \ldots$

Proof. We prove the " $\Rightarrow$ " direction in the Appendix and omit the easier " $\Leftarrow$ " direction.

One additional property of the construction will be useful in the following:

Lemma $3.4 E_{n}$ and $F_{n}$ have no infinite run, regardless of the initial configuration.

Proof. We first deal with the $E_{n}$ 's by induction over $n$. $E_{2}$ terminates since its loop consumes from $c_{2}$. For $n>2$, a run of $E_{n}$ cannot visit $q_{n}$ infinitely often (this would consume infinitely from $c_{n}$ ) and, by ind. hyp., cannot contain an infinite subrun of $E_{n-1}$ (obviously, the $T_{n-1}$ part must terminate too).

For the $F_{n}$ 's, we first observe (again using induction on $n$ ) that, if $c_{1}$ contains at least one 1 , then a run from $s_{n}^{\prime}$ to $f_{n}^{\prime}$ removes strictly more 1's than it writes back. Finally, traversing $T_{n-1}$ must move some 1's to $c_{1}$ (or lose them).

\section{The hardness results}

Expander and folder systems can now be used to prove Theorem 2.3.

\subsection{Hardness for reachability}

Let's consider a Turing Machine (a TM) $M$ that is started on a blank work tape of size $m$ (for some $m$ ) and that never goes beyond the allocated workspace. One can build a channel system $S$ that simulates $M$ using as workspace channel $c_{1}$ initially filled with $\mathrm{b} 1^{m} \mathrm{e}$. We do not describe further the construction of $S$ since it follows the standard simulation of TM's by channel systems (from [BZ83]) ${ }^{3}$. Since $M$ never goes beyond the allocated workspace, the

\footnotetext{
${ }^{3}$ And since TM's are not really required and could be replaced by perfect channel systems that preserve the size of the channel contents.
} 
transition rules of $S$ always write exactly as many messages as they read, so that if no loss occurs the channel always contains the same number of letters. The resulting system has two types of runs: perfect runs where $M$ is simulated faithfully, and lossy runs that do not really simulate $M$ but where messages have been irremediably lost.

Now, in order to know whether $M$ accepts in space $m$, there only remains to provide $S$ with enough workspace, and to look at runs where no message is lost. This is exactly what is done by $S_{M}^{n}$, depicted in Fig. 6, where expander $E_{n}$ provides a potentially large $\lceil m\rceil$ in channel $c_{1}$ and folder $F_{n}$ is used to check that no message has been lost.

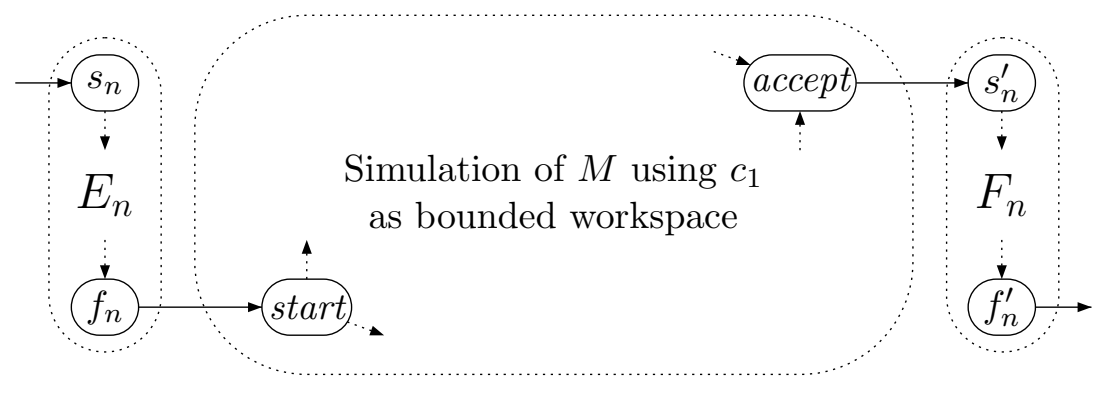

Figure 6: $S_{M}^{n}$ : simulating Turing machine $M$ with huge workspace

Thus any run of $S_{M}^{n}$ of the form $\left\langle s_{n},\lceil 0\rceil, \ldots,\lceil 0\rceil,\lceil 2\rceil\right\rangle \stackrel{*}{\rightarrow}\left\langle f_{n}^{\prime},\lceil 0\rceil, \ldots,\lceil 0\rceil,\lceil 2\rceil\right\rangle$ is perfect and must visit both $\langle$ start $,\lceil A c k(n)\rceil,\lceil 0\rceil, \ldots,\lceil 0\rceil\rangle$ and $\langle\operatorname{accept},\lceil\operatorname{Ack}(n)\rceil,\lceil 0\rceil, \ldots,\lceil 0\rceil\rangle$. Hence

Proposition $4.1\left\langle s_{n},\lceil 0\rceil, \ldots,\lceil 0\rceil,\lceil 2\rceil\right\rangle \stackrel{*}{\rightarrow}\left\langle f_{n}^{\prime},\lceil 0\rceil, \ldots,\lceil 0\rceil,\lceil 2\rceil\right\rangle$ in $S_{M}^{n}$ iff $M$ accepts in space $\operatorname{Ack}(n)$.

Therefore, since $S_{M}^{n}$ has size $O(n+|M|)$, reachability for lossy channel systems is at least as hard as termination for TM's running in Ackermann space. Hence

Corollary 4.2 Reachability for lossy channel systems has nonprimitive recursive complexity.

\subsection{Hardness for termination}

The second hardness result uses a slight adaptation of our previous construction, and relies on the following simulation (Fig. 7).

Here $S_{M}^{\prime n}$ fills two channels with $\lceil A c k(n)\rceil: c_{1}$ used as before as working space, and $c_{0}$ used as a countdown that ensures termination of the simulation of $M$. Every time one step of $M$ is simulated, $S^{\prime n}{ }_{M}$ consumes one 1 from $c_{0}$. When the accepting state of $M$ is reached, $S_{M}^{\prime n}$ moves to $s_{n}^{\prime}$ where it uses $F_{n}$ to check that $c_{1}$ does contain $\lceil A c k(n)\rceil$ (i.e. the simulation was faithful). If the check succeeds, $S^{\prime n}{ }_{M}$ can enter a loop. Therefore, a run of $S^{\prime n}$ terminates when the simulation is not perfect, or when $M$ does not accept in at most $A c k(n)$ steps.

Proposition $4.3 S^{\prime n}$ has an infinite run from $\left\langle s_{n},\lceil 0\rceil, \ldots,\lceil 0\rceil,\lceil 2\rceil\right\rangle$ iff $M$ accepts in time $\operatorname{Ack}(n)$.

Proof. Clearly, $S^{\prime n}$ can only reach the final loop if the simulation is faithful, and halts in at most $A c k(n)$ steps. There remains to show that the unfaithful, lossy, behaviors are terminating: Lemma 3.4 deals with the $E_{n}$ and $F_{n}$ part of $S_{M}^{\prime n}$, the duplication gadget (between $f_{n}$ and start) obviously terminates, and we solve the problem for the simulation part by programming it in such a way that the rotation of the tape (necessary for simulating a 


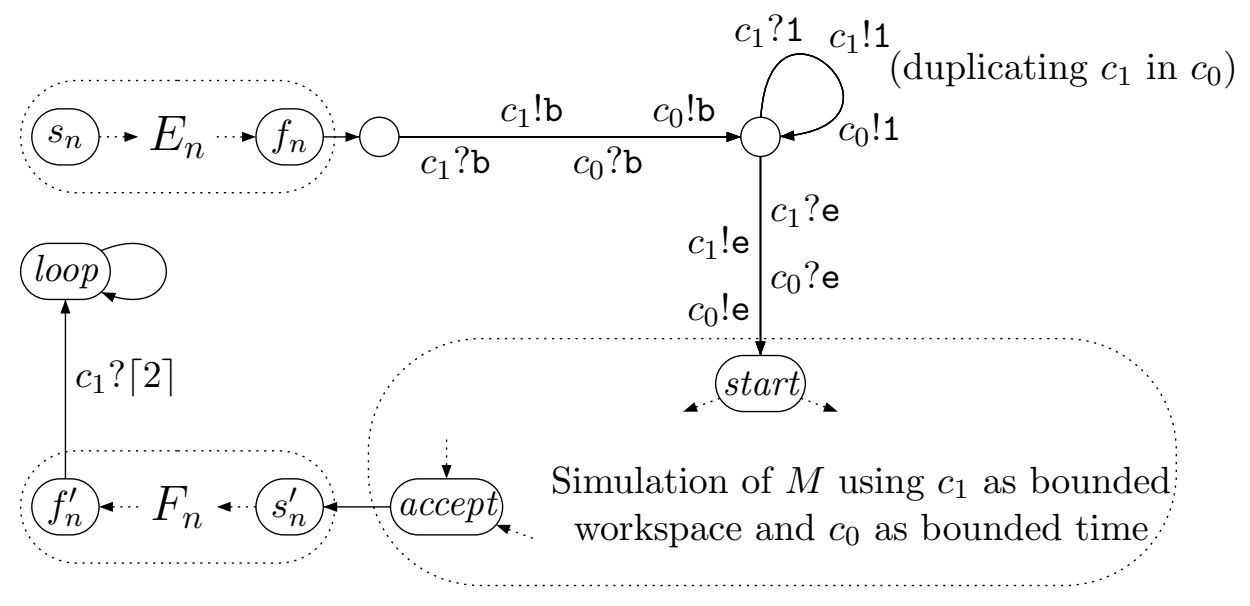

Figure 7: $S_{M}^{\prime n}$ : another simulation of $M$ with huge workspace

TM) cannot induce non-termination. One way ${ }^{4}$ to achieve this is to use two copies (one positive and one negative) of the TM alphabet: in "+" mode, the simulation reads +-letters and writes back their --twin. Only when an actual TM step is performed does $S_{M}^{\prime n}$ swap from "+" mode to "-" mode and vice versa. More details can be found in section 5 where the same trick is used.

This shows that termination for lossy channel systems is at least as hard as termination for TM's runnings in Ackermann time. Hence

Corollary 4.4 Termination for lossy channel systems has nonprimitive recursive complexity.

\section{$5 \quad$ Systems with only one channel}

Our construction used several channels for clarity, not out of necessity, and our result still holds when we restrict ourselves to lossy channel systems with only one channel. This is one more application of the slogan "lossy systems with $k$ channels can be encoded into lossy systems with one channel". The encoding given in [AJ96a, Section 4.5] preserves the existence of runs that visit a given control state infinitely often. Below we give another encoding that further preserves termination and reachability. It uses standard techniques (e.g. from the study of TM's with $k$ tapes) and the only original aspect is the lossy behavior of our systems.

Consider a system $S=\langle Q, C, \Sigma, \Delta\rangle$ that uses channels $c_{1}, \ldots, c_{k}$. We simulate $S$ by a system $S^{\prime}=\left\langle Q^{\prime},\{c\}, \Sigma^{\prime}, \Delta^{\prime}\right\rangle$ that uses one single channel $c$. Without loss of generality we assume a different subalphabet is used with every channel of $S$ (i.e. $\Sigma$ is partitioned in disjoint alphabets $\left.\Sigma_{1} \cup \cdots \cup \Sigma_{k}\right)$. The encoding uses a larger alphabet where $k$ markers $\#_{1}, \ldots, \#_{k}$ have been added, and where every letter comes in two copies (a positive and a negative one). Formally $\Sigma^{\prime} \stackrel{\text { def }}{=}\left(\Sigma \cup\left\{\#_{1}, \ldots, \#_{k}\right\}\right) \times\{+,-\}$ and a pair $(x, \alpha) \in \Sigma^{\prime}$ is written shortly $x^{\alpha}$. For $x \in \Sigma_{i}$, an occurrence of some $x^{\alpha}$ in $c$ means "one $x$ in $c_{i}$ " (and the polarity $\alpha$ is only used for bookkeeping purposes).

\footnotetext{
${ }^{4}$ An alternative solution would use $c_{0}$ as a countdown for channel system steps rather than TM steps, but Prop. 4.3 would have to be reworded in a clumsy way.
} 
A $k$-tuple $\left\langle w_{1}, \ldots, w_{k}\right\rangle \in \Sigma_{1}^{*} \times \cdots \times \Sigma_{k}^{*}$ is encoded as $\left(\#_{1} w_{1} \#_{2} w_{2} \ldots \#_{k} w_{k}\right)^{\alpha}$ where a same polarity $\alpha$ is used to label all letters. For example, $\langle a b, \varepsilon, \mathrm{ddc}\rangle$ is coded as $U=$ $\#_{1}^{+} \mathrm{a}^{+} \mathrm{b}^{+} \#_{2}^{+} \#_{3}^{+} \mathrm{d}^{+} \mathrm{d}^{+} \mathrm{c}^{+}$under positive polarity.

Fig. 8 shows how two example rules from $\Delta$ (left-hand side) are encoded in $S^{\prime}$ (right-hand side). On this figure, four loops (marked by $*$ ) provide for the rotation of the contents of $c$
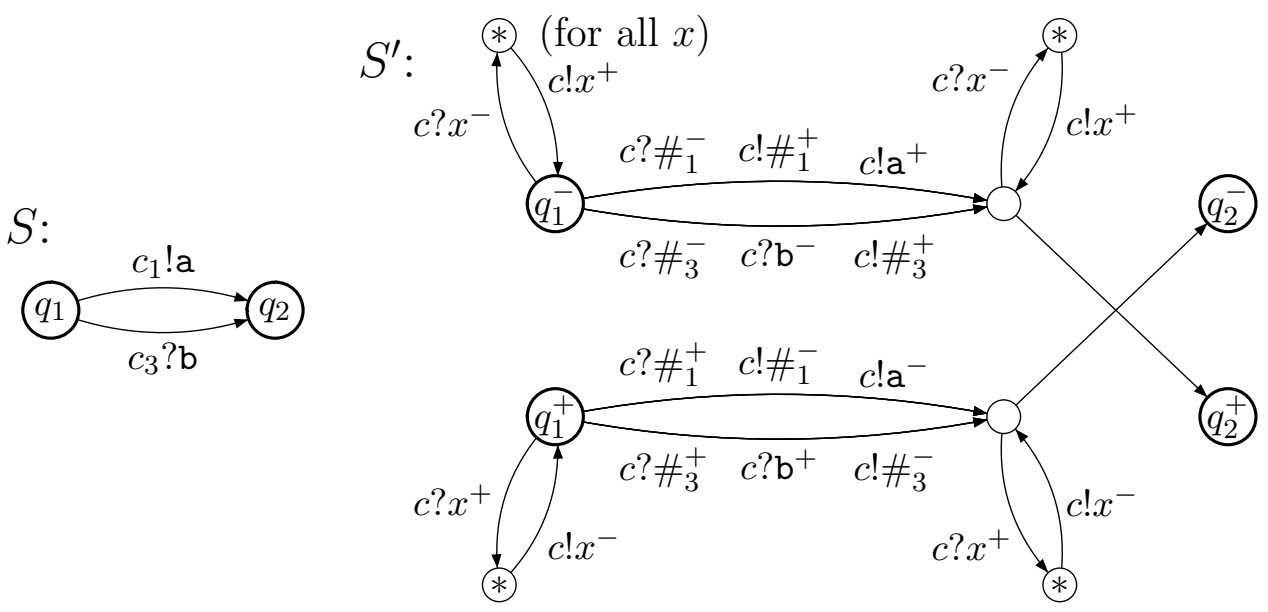

Figure 8: From $S$ to $S^{\prime}$ : encoding $k$ channels into one

(with a change of polarity): they are shorthands for several loops as indicated by the "for all $x$ " comment. A state $q$ from $S$ gives rise to two copies $q^{+}$and $q^{-}$in $S^{\prime}: q^{+}$reads positive letters and writes negative letters, thus preventing non-termination induced by the rotation loops, $q^{-}$does the converse, and $S^{\prime}$ changes polarity each time a step of $S$ has been simulated.

Now, if $W$ and $V$ are encodings of $\left\langle w_{1}, \ldots, w_{k}\right\rangle$ and $\left\langle v_{1}, \ldots, v_{k}\right\rangle$, if $\alpha \in\{+,-\}$, we have $\left\langle q, w_{1}, \ldots, w_{k}\right\rangle \stackrel{*}{\rightarrow}_{\text {perf }}\left\langle q^{\prime}, v_{1}, \ldots, v_{k}\right\rangle$ (in $S$ ) iff $\left\langle q^{\alpha}, W^{\alpha}\right\rangle \stackrel{*}{\rightarrow}_{\text {perf }}\left\langle q^{\prime \beta}, V^{\beta}\right\rangle$ for some $\beta \in\{+,-\}$. This extends to lossy behaviors:

$$
\begin{aligned}
\left\langle q, w_{1}, \ldots, w_{k}\right\rangle \stackrel{*}{\rightarrow}\left\langle q^{\prime}, v_{1}, \ldots, v_{k}\right\rangle & \text { iff }\left\langle q^{\alpha}, W^{\alpha}\right\rangle \stackrel{*}{\rightarrow}\left\langle q^{\prime \beta}, V^{\beta}\right\rangle \text { for some } \beta, \\
S \text { terminates from }\left\langle q, w_{1}, \ldots, w_{k}\right\rangle & \text { iff } S^{\prime} \text { terminates from }\left\langle q^{\alpha}, W^{\alpha}\right\rangle .
\end{aligned}
$$

Note that these equivalences only hold for $W$ and $V$ that are correct encodings of $k$-tuples, and for $q, q^{\prime}$ that are original states from $Q . S^{\prime}$ has behaviors that do not correspond to behaviors of $S$ in the sense of (12), e.g. when it gets blocked in new states, or when it loses one of the $\#_{i}$ markers.

Corollary 5.1 Reachability and termination for lossy single-channel systems have nonprimitive recursive complexity.

\section{Conclusion}

There exist several constructions in the literature where a problem $P$ is shown undecidable for lossy channel systems by simulating a Turing machine in such a way that the faithfulness of the simulation can be ensured, or checked, or rewarded in some way. Our construction uses similar tricks since it first builds a nonprimitive recursive number of messages, and later checks that none has been lost. Still, there are a number of new aspects in our construction, 
and this explains why it is the first complexity result for decidable problems on lossy channel systems.

\section{Acknowledgements}

We are grateful to Alain Finkel who brought this topic to our attention, to Petr Jančar who suggested major improvements on an earlier draft, and to the anonymous referee who helped us improve section 5 .

\section{A Appendix: Proof of Prop. 3.2}

The difficulty with the " $\Rightarrow$ " direction of Prop. 3.2 is that we have to consider lossy behaviors that need not respect the logic of the $E_{n}$ systems we designed. However, when we restrict our attention to behaviors that do not lose the $\mathrm{b}$ and e markers, managing the problem becomes feasible.

We start with the following lemma:

Lemma A.1 If a run $\left\langle s_{n},\left\lceil a_{1}\right\rceil, \ldots,\left\lceil a_{n}\right\rceil\right\rangle \stackrel{*}{\rightarrow}\left\langle f_{n}, w_{1}, \ldots, w_{n}\right\rangle$ of $E_{n}$ is such that every $w_{i}$ contains one $\mathrm{b}$ and one $\mathrm{e}$, then every $w_{i}$ has the form $\mathrm{b} 1^{*} \mathrm{e}$, i.e. encodes a number.

The same holds for a run $\left\langle t_{n},\left\lceil a_{1}\right\rceil, \ldots,\left\lceil a_{n}\right\rceil\right\rangle \stackrel{*}{\rightarrow}\left\langle x_{n}, w_{1}, \ldots, w_{n}\right\rangle$ of $T_{n}$.

Proof. Since our systems always write a $\mathrm{b}$ or a e after they consumed one, saying that every $w_{i}$ contains one $\mathrm{b}$ and one e means that losses did not concern the markers. Therefore, it only remains to prove that the pattern $\mathrm{b} 1^{*} \mathrm{e}$ is respected, even when some 1 's have been lost.

$E_{2}$ has to consume all of $\left\lceil a_{1}\right\rceil$ and $\left\lceil a_{2}\right\rceil$ and what it writes in $c_{1}$ and $c_{2}$ encodes a number even after losses. The same reasoning applies to the channels $c_{1}$ and $c_{n}$ of $T_{n}$, while the other channels are untouched (and losses there respect the $\mathrm{b} 1^{*} \mathrm{e}$ pattern).

For $E_{n}$ with $n>2$, we proceed by induction over $n$. For $c_{n}$, observe that all of $a_{n}$ is consumed and replaced by $\lceil 0\rceil$. For the other channels $\left(c_{1}\right.$ to $\left.c_{n-1}\right)$, they all contain a number when the run first reaches $q_{n}$ and, since the lemma holds for $T_{n-1}$ and $E_{n-1}$ (by ind. hyp.), this remains the case every time the run revisits $q_{n}$, until it eventually reaches $f_{n}$. Then all channels contain encodings of numbers.

We are now ready to prove that

$$
\left\langle s_{n},\lceil 0\rceil, \ldots,\lceil 0\rceil,\lceil k\rceil\right\rangle \stackrel{*}{\rightarrow}\left\langle f_{n},\left\lceil a_{1}\right\rceil, \ldots,\left\lceil a_{n}\right\rceil\right\rangle \Rightarrow\left\{\begin{array}{l}
a_{1} \leq A_{n}(k) \text {, and } \\
a_{2}=\ldots=a_{n}=0 .
\end{array}\right.
$$

by induction over $n$. The base case $n=2$ is left to the reader: a simple inspection of $E_{2}$ shows it weakly computes $A_{2}(k)=2 k$.

For $n>2$ we consider a run

$$
\left\langle s_{n},\lceil 0\rceil, \ldots,\lceil 0\rceil,\lceil k\rceil\right\rangle \stackrel{*}{\rightarrow}\left\langle f_{n},\left\lceil a_{1}\right\rceil, \ldots,\left\lceil a_{n}\right\rceil\right\rangle
$$

and isolate the configurations where (14) visits $q_{n}$ and $f_{n-1}$ by writing it under the form

$$
\begin{aligned}
&\left\langle s_{n},\lceil 0\rceil, \ldots,\lceil k\rceil\right\rangle \stackrel{*}{\rightarrow}\left\langle q_{n}, w_{1}^{0}, \ldots, w_{n}^{0}\right\rangle \stackrel{*}{\rightarrow}\left\langle f_{n-1}, v_{1}^{1}, \ldots, v_{n}^{1}\right\rangle \\
& \rightarrow\left\langle q_{n}, w_{1}^{1}, \ldots, w_{n}^{1}\right\rangle \stackrel{*}{\rightarrow}\left\langle f_{n-1}, v_{1}^{2}, \ldots, v_{n}^{2}\right\rangle \rightarrow \cdots \stackrel{*}{\rightarrow}\left\langle f_{n-1}, v_{1}^{m}, \ldots, v_{n}^{m}\right\rangle \\
& \rightarrow\left\langle q_{n}, w_{1}^{m}, \ldots, w_{n}^{m}\right\rangle \stackrel{*}{\rightarrow}\left\langle f_{n},\left\lceil a_{1}\right\rceil, \ldots,\left\lceil a_{n}\right\rceil\right\rangle .
\end{aligned}
$$


Since the $\mathrm{b}$ and e markers are not lost in (14), we can state that all $w_{n}^{i}$ and $v_{n}^{i}$ have the form $1^{k_{i}} \mathrm{eb}$ (resp. $1^{k_{i}^{\prime}} \mathrm{eb}$ ). Since the transition leaving $q_{n}$ for $t_{n-1}$ consumes one 1 from $c_{n}$, one sees that

$$
k \geq k_{0}>k_{1}^{\prime} \geq k_{1}>k_{2}^{\prime} \geq k_{2}>\cdots \geq k_{m},
$$

implying $m \leq k$. Finally $a_{n}=0$ (since $f_{n}$ can only be reached by consuming e from $c_{n}$ ) and this concludes the proof for the part that concerns $c_{n}$.

When we consider the other channels $\left(c_{1}\right.$ to $\left.c_{n-1}\right)$ the proof of Lemma A.1 shows that, for $i<n$, the $w_{i}^{j}$ and $v_{i}^{j}$ all are encodings of numbers.

Furthermore, they satisfy

$$
\begin{aligned}
& w_{1}^{j} \sqsubseteq\lceil\underbrace{A_{n-1} \circ \cdots A_{n-1}}_{j \text { times }}(1)\rceil \quad \text { and } \quad w_{i}^{j}=\lceil 0\rceil \text { if } 2 \leq i \leq n-1 \\
& v_{n-1}^{j} \sqsubseteq w_{1}^{j-1} \quad \text { and } \quad v_{i}^{j}=\lceil 0\rceil \text { if } 1 \leq i \leq n-2 .
\end{aligned}
$$

as we prove by induction on $j$. The base case, $\left(\mathrm{W}_{0}\right)$ is a consequence of the assumption (14). Then one shows that $\left(\mathrm{W}_{j}\right)$ entails $\left(\mathrm{V}_{j+1}\right)$ using Prop. 3.1. Finally, one proves that $\left(\mathrm{V}_{j}\right)$ and $\left(\mathrm{W}_{j-1}\right)$ entail $\left(\mathrm{W}_{j}\right)$ using $\left(\mathrm{H}_{n-1}\right)$.

One concludes the proof of $\left(\mathrm{H}_{n}\right)$ by observing that $m \leq k$ and $\left(\mathrm{W}_{m}\right)$ entail the right hand side of $\left(\mathrm{H}_{n}\right)$ because of the monotonicity and expansion properties of $A_{n}$ stated in (5).

\section{B Appendix: Proof of Prop. 3.3}

For the " $\Rightarrow$ " direction, we proceed as with the proof of Prop. 3.2, and start with a result mimicking Lemma A.1:

Lemma B.1 If a run $\left\langle s_{n}^{\prime},\left\lceil a_{1}\right\rceil, \ldots,\left\lceil a_{n}\right\rceil\right\rangle \stackrel{*}{\rightarrow}\left\langle f_{n}^{\prime}, w_{1}, \ldots, w_{n}\right\rangle$ of $F_{n}$ is such that every $w_{i}$ contains one $\mathrm{b}$ and one $\mathrm{e}$, then every $w_{i}$ encodes a number.

Proof. Omitted.

We now prove

$$
\left\langle s_{n}^{\prime},\lceil m\rceil,\lceil 0\rceil, \ldots,\lceil 0\rceil\right\rangle \stackrel{*}{\rightarrow}\left\langle f_{n}^{\prime},\left\lceil a_{1}\right\rceil, \ldots,\left\lceil a_{n}\right\rceil\right\rangle \Rightarrow\left\{\begin{array}{l}
A_{n}\left(a_{n}\right) \leq m, \text { and } \\
a_{1}=\ldots=a_{n-1}=0 .
\end{array}\right.
$$

by induction over $n$. The base $n=2$ is easy to see. For $n>2$, we consider a run $\left\langle s_{n}^{\prime},\lceil m\rceil,\lceil 0\rceil, \ldots,\lceil 0\rceil\right\rangle \stackrel{*}{\rightarrow}\left\langle f_{n}^{\prime},\left\lceil a_{1}\right\rceil, \ldots,\left\lceil a_{n}\right\rceil\right\rangle$ and isolate the configurations where it visits $q_{n}^{\prime}$ and $f_{n-1}^{\prime}$ by writing it under the form

$$
\begin{aligned}
\left\langle s_{n}^{\prime},\lceil m\rceil, \ldots,\lceil 0\rceil\right\rangle \stackrel{*}{\rightarrow}\left\langle q_{n}^{\prime}, w_{1}^{0}, \ldots, w_{n}^{0}\right\rangle \stackrel{*}{\rightarrow}\left\langle f_{n-1}^{\prime}, v_{1}^{1}, \ldots, v_{n}^{1}\right\rangle \\
\quad \stackrel{*}{\rightarrow}\left\langle q_{n}^{\prime}, w_{1}^{1}, \ldots, w_{n}^{1}\right\rangle \stackrel{*}{\rightarrow}\left\langle f_{n-1}^{\prime}, v_{1}^{2}, \ldots, v_{n}^{2}\right\rangle \stackrel{*}{\rightarrow} \cdots \stackrel{*}{\rightarrow}\left\langle f_{n-1}^{\prime}, v_{1}^{l}, \ldots, v_{n}^{l}\right\rangle \\
\quad \stackrel{*}{\rightarrow}\left\langle q_{n}^{\prime}, w_{1}^{l}, \ldots, w_{n}^{l}\right\rangle \stackrel{*}{\rightarrow}\left\langle f_{n}^{\prime},\left\lceil a_{1}\right\rceil, \ldots,\left\lceil a_{n}\right\rceil\right\rangle .
\end{aligned}
$$

We start with the contents of $c_{n}$ : the $w_{n}^{i}$ and $v_{n}^{i}$ have the form eb1 ${ }^{k_{i}}$ and, resp., eb1 ${ }^{k_{i}^{\prime}}$. The transition from $q_{n}^{\prime}$ to $s_{n-1}^{\prime}$ writes one 1 to $c_{n}$, so that, for $i=1, \ldots, l, k_{i} \leq k_{i}^{\prime} \leq 1+k_{i-1}$. With $k_{0}=0$ and $k_{l} \geq a_{n}$ we deduce $l \geq a_{n}$. 
When we consider the contents of the other channels, the $w_{i}^{j}$ 's and $v_{i}^{j}$ 's encode numbers for $j=1, \ldots, n-1$ (by Lemma B.1). Using $\left(\mathrm{H}_{n-1}^{\prime}\right)$ one shows, by induction on $i$, that $w_{i}^{j}=\lceil 0\rceil$ for $j=2, \ldots, n-1$, and $v_{i}^{j}=\lceil 0\rceil$ for $j=1, \ldots, n-2$, so that $a_{1}=\ldots=a_{n-1}=0$. Then, writing $w_{i}^{1}=\left\lceil m_{i}\right\rceil$ and $v_{i}^{n-1}=\left\lceil x_{i}\right\rceil,\left(\mathrm{H}_{n-1}^{\prime}\right)$ further entails

$$
\begin{aligned}
A_{n-1}\left(x_{1}\right) & \leq m_{0}, & A_{n-1}\left(x_{2}\right) \leq m_{1} & \leq x_{1}, \\
A_{n-1}\left(x_{l}\right) & \leq m_{l-1} \leq x_{l-1}, & m_{l} & \leq x_{l} .
\end{aligned}
$$

so that, by monotonicity of $A_{n-1}$,

$$
\underbrace{A_{n-1} \circ \cdots A_{n-1}}_{l \text { times }}\left(m_{l}\right) \leq m_{0} .
$$

Since $1 \leq m_{l}$ (because finally leaving $q_{n}^{\prime}$ consumes $\lceil 1\rceil$ from $c_{n-1}$ ), $m_{0} \leq m, a_{n} \leq l$ and (5) entail that $A_{n}\left(a_{n}\right) \leq m$, completing the proof.

\section{References}

[AAB99] P. A. Abdulla, A. Annichini, and A. Bouajjani. Symbolic verification of lossy channel systems: Application to the bounded retransmission protocol. In Proc. 5th Int. Conf. Tools and Algorithms for the Construction and Analysis of Systems (TACAS'99), Amsterdam, The Netherlands, Mar. 1999, volume 1579 of Lecture Notes in Computer Science, pages 208-222. Springer, 1999.

[ABJ98] P. A. Abdulla, A. Bouajjani, and B. Jonsson. On-the-fly analysis of systems with unbounded, lossy FIFO channels. In Proc. 10th Int. Conf. Computer Aided Verification (CAV'98), Vancouver, BC, Canada, June-July 1998, volume 1427 of Lecture Notes in Computer Science, pages 305-318. Springer, 1998.

[ABPJ00] P. A. Abdulla, C. Baier, S. Purushothaman Iyer, and B. Jonsson. Reasoning about probabilistic lossy channel systems. In Proc. 11th Int. Conf. Concurrency Theory (CONCUR'2000), University Park, PA, USA, Aug. 2000, volume 1877 of Lecture Notes in Computer Science, pages 320-333. Springer, 2000.

[AČJT00] P. A. Abdulla, K. Čerāns, B. Jonsson, and Yih-Kuen Tsay. Algorithmic analysis of programs with well quasi-ordered domains. Information and Computation, 160(1/2):109-127, 2000.

[AJ96a] P. A. Abdulla and B. Jonsson. Undecidable verification problems for programs with unreliable channels. Information and Computation, 130(1):71-90, 1996.

[AJ96b] P. A. Abdulla and B. Jonsson. Verifying programs with unreliable channels. Information and Computation, 127(2):91-101, 1996.

[AK95] P. A. Abdulla and M. Kindahl. Decidability of simulation and bisimulation between lossy channel systems and finite state systems. In Proc. 6th Int. Conf. Theory of Concurrency (CONCUR'95), Philadelphia, PA, USA, Aug. 1995, volume 962 of Lecture Notes in Computer Science, pages 333-347. Springer, 1995. 
[AKP97] P. A. Abdulla, M. Kindahl, and D. Peled. An improved search strategy for lossy channel systems. In Proc. Joint Int. Conf. Formal Description Techniques and Protocol Specification, Testing, and Verification (FORTE/PSTV'97), Osaka, Japan, Nov. 1997, pages 251-264. Chapman \& Hall, 1997.

[Boc78] G. von Bochmann. Finite state description of communication protocols. Computer Networks and ISDN Systems, 2:361-372, 1978.

[BZ83] D. Brand and P. Zafiropulo. On communicating finite-state machines. Journal of the ACM, 30(2):323-342, 1983.

[CFP96] G. Cécé, A. Finkel, and S. Purushothaman Iyer. Unreliable channels are easier to verify than perfect channels. Information and Computation, 124(1):20-31, 1996.

[DFS98] C. Dufourd, A. Finkel, and Ph. Schnoebelen. Reset nets between decidability and undecidability. In Proc. 25th Int. Coll. Automata, Languages, and Programming (ICALP'98), Aalborg, Denmark, July 1998, volume 1443 of Lecture Notes in Computer Science, pages 103-115. Springer, 1998.

[DJS99] C. Dufourd, P. Jančar, and Ph. Schnoebelen. Boundedness of Reset P/T nets. In Proc. 26th Int. Coll. Automata, Languages, and Programming (ICALP'99), Prague, Czech Republic, July 1999, volume 1644 of Lecture Notes in Computer Science, pages 301-310. Springer, 1999.

[Fin94] A. Finkel. Decidability of the termination problem for completely specificied protocols. Distributed Computing, 7(3):129-135, 1994.

[FS01] A. Finkel and Ph. Schnoebelen. Well structured transition systems everywhere! Theoretical Computer Science, 256(1-2):63-92, 2001.

[Hac76] M. Hack. The equality problem for vector addition systems is undecidable. Theoretical Computer Science, 2(1):77-95, 1976.

[Hig52] G. Higman. Ordering by divisibility in abstract algebras. Proc. London Math. Soc. (3), 2(7):326-336, 1952.

[Jan01] P. Jančar. Nonprimitive recursive complexity and undecidability for Petri net equivalences. Theoretical Computer Science, 256(1-2):23-30, 2001.

[May00] R. Mayr. Undecidable problems in unreliable computations. In Proc. 4th Latin American Symposium on Theoretical Informatics (LATIN'2000), Punta del Este, Uruguay, Apr. 2000, volume 1776 of Lecture Notes in Computer Science, pages 377-386. Springer, 2000.

[MM81] E. W. Mayr and A. R. Meyer. The complexity of the finite containment problem for Petri nets. Journal of the ACM, 28(3):561-576, 1981.

[Sch01] Ph. Schnoebelen. Bisimulation and other undecidable equivalences for lossy channel systems. In Proc. 4th Int. Symp. Theoretical Aspects of Computer Software (TACS'2001), Sendai, Japan, Oct. 2001, volume 2215 of Lecture Notes in Computer Science, pages 385-399. Springer, 2001. 